\title{
Electrical Stimulation Electrode Type
}

National Cancer Institute

\section{Source}

National Cancer Institute. Electrical Stimulation Electrode Type. NCI Thesaurus. Code C114145.

The type of electrode used on the subject during electrical stimulation of the body or organ. 